\title{
Evaluation of the targeted delivery of 5-fluorouracil and ascorbic acid into the brain with ultrasound-responsive nanobubbles
}

Yusuke Miura ${ }^{1 \#}$, Yuki Fuchigami ${ }^{1 \#}$, Masayori Hagimori ${ }^{1}$, Hiroki Sato ${ }^{1}$, Koki Ogawa $^{1}$, Chie Munakata ${ }^{1}$, Mitsuhiro Wada ${ }^{1 a}$, Kazuo Maruyama ${ }^{2}$ and Shigeru Kawakami ${ }^{1 *}$

${ }^{1}$ Department of Pharmaceutical Informatics, Graduate School of Biomedical Sciences, Nagasaki University, 1-7-1 Sakamoto, Nagasaki-shi, Nagasaki 852-8588, Japan

${ }^{2}$ Laboratory of Drug Delivery System, Faculty of Pharma-Sciences, Teikyo University, 2-11-1 Kaga, Itabashi-ku, Tokyo 173-8605, Japan

${ }^{a}$ Present address: School of Pharmaceutical Sciences, Kyushu University of Health and Welfare, 1714-1 Yoshino-machi, Nobeoka-shi, Miyazaki 882-8508, Japan

${ }^{\#}$ These authors equally contributed to this work.

The authors report no conflicts of interest

Provide full correspondence details here including e-mail for the * corresponding author E-mail: skawakam@nagasaki-u.ac.jp (S. Kawakami)

Phone: +81-95-819-8563

Fax: +81-95-819-8563 


\section{Evaluation of the targeted delivery of 5-fluorouracil and ascorbic acid into the brain with ultrasound-responsive nanobubbles}

Recently, ultrasound-induced drug delivery into the brain using bubble formulations has been developed. After the brain delivery, however, the information on pharmacokinetics of hydrophilic drugs in the brain is lacking. In this study, to clarify the time-course pharmacokinetics of hydrophilic drugs, we used a brain microdialysis method. Using ultrasound-responsive nanobubbles (bubble liposomes [BLs]) with ultrasound irradiation, two hydrophilic drugs, 5fluorouracil (5-FU) and ascorbic acid, were delivered into the brain of mice and rats, and their time-course pharmacokinetics were evaluated with microdialysis. The results indicated that the time-course pharmacodynamics of ascorbic acid evaluated by examining its antioxidant capacity supported the time-course pharmacokinetics. Additionally, to strengthen the evidences of our evaluation, we varied the effect of BLs dose, and duration and intensity of ultrasound irradiation on drug delivery. Among them, when the dose of BLs was changed, the trend of 5-FU intracerebral migration was consistent with other report. In conclusion, we succeeded in clarifying the time-course pharmacokinetics of the two hydrophilic drugs after the brain delivery with bubble formulations and ultrasound irradiation using mice and rats.

Keywords: drug delivery system; ultrasound; bubble liposomes; blood-brain barrier; brain microdialysis 


\section{Introduction}

Recently, targeted delivery using an external stimuli-responsive drug delivery system (DDS) has attracted attention. Ultrasound is a type of external energy that is widely used for medical diagnostics, can be applied for clinical uses, and safely reaches the deeper tissues of the human body [1]. In the field of ultrasound-responsive DDS, the targeted delivery into the brain using bubble formulations and ultrasound irradiation has been reported to be successful because of disruption of the blood-brain barrier (BBB) [2-6]. By using ultrasound-responsive bubble formulations, animals were treated with hydrophilic drugs or genes. Among the studies, microbubbles were used for the brain delivery of doxorubicin [7] and liposomal doxorubicin [8]. More recently, Negishi et al. have used nanobubbles (bubble liposomes [BLs]) to reach deeper regions of the brain, and showed that plasmid DNA was delivered into the brain with BLs and high intensity focused ultrasound (HIFU) irradiation [9]. In the field of ultrasound-induced drug delivery into the brain, however, the information on pharmacokinetics of hydrophilic drugs in the brain is lacking. To develop this DDS to the clinical applications, it is necessary to evaluate the pharmacokinetics of drugs themselves in the brain. Therefore, the evaluation systems for detailed pharmacokinetics of hydrophilic drugs in the brain after the brain delivery are required.

Brain microdialysis can be used to evaluate the time-course pharmacokinetics of hydrophilic agents in the brain $[10,11]$. Yang et al. have demonstrated the time-course pharmacokinetics of boronophenylalanine (BPA), an agent for boron neutron capture therapy, in the rat brain after administration of microbubbles with focused ultrasound irradiation using microdialysis [12]. They have shown that microdialysis is useful for the assessment of the pharmacokinetics of BPA in the brain. This is the only study that evaluated the time-course pharmacokinetics of hydrophilic agents after the brain delivery using microdialysis, but BPA alone does not affect the brain cells. Therefore, it 
is necessary to evaluate the detailed time-course pharmacokinetics of more drugs which affect the brain alone, focusing on hydrophilic drugs which is expected to be prevented migrating across the $\mathrm{BBB}$.

Our group has been developing DDS for liver [13], spleen [14], solid tumours $[15,16]$, and peritoneal tissues [17] using ultrasound-responsive bubble formulations. Additionally, we have been studying the pharmacological and neurochemical effects using a brain microdialysis method [18]. Combining ultrasound-induced drug delivery into the brain with microdialysis, the pharmacokinetics of hydrophilic drugs in the brain must become clear. Because the brain microdialysis is an established technique, it should be possible to easily apply to the evaluation for ultrasound-induced drug delivery into the brain.

In the present study, we examined ultrasound-induced drug delivery into the brain with BLs using a brain microdialysis method. We selected two hydrophilic drugs, 5-fluorouracil (5-FU) and ascorbic acid, as models for evaluation of targeted drug delivery into the brain because hydrophilic drugs can also be recovered by a microdialysis probe. By collecting dialysates over specific intervals, we evaluated the time-course pharmacokinetics of 5-FU and ascorbic acid in the brain of mice and rats. We also evaluated the time-course pharmacodynamics of ascorbic acid by measuring the antioxidant capacity of the dialysates from the rat brain using a semi-micro flow injection analysis (SMFIA) method [19] to support the rat brain pharmacokinetics. We clarified that ascorbic acid was not distributed into the brain at our experimental condition [19]; therefore ascorbic acid was selected as a model drug for targeted brain delivery in this study. Additionally, we varied the effect of BLs and ultrasound on 5-FU delivery into the brain in our conditions using microdialysis in mice, observing that the trend of 5-FU intracerebral migration is consistent with other reports. As part of the 
evaluation, we varied the dose of BLs, the duration of ultrasound irradiation and the ultrasound intensity.

\section{Methods}

\section{Animals}

Male ddY mice (24-31 g) were purchased from Kiwa Laboratory Animals Co., Ltd. (Wakayama, Japan) and male Wistar rats (255-290 g) were purchased from CLEA Japan, Inc. (Tokyo, Japan). All the animals had free access to food and water. The animal room was air-conditioned and maintained with a 12-hour light-dark cycle. All the experiments with animals were performed under the Regulations of the Animal Care and Use Committee of Nagasaki University.

\section{Preparation of drugs and BLS}

5-FU (Tokyo Chemical Industry Co., Ltd., Tokyo, Japan) was dissolved in saline at $10 \mathrm{mg} / \mathrm{mL}$. L-Ascorbic acid was purchased from Wako Pure Chemical Industries, Ltd. (Osaka, Japan) and dissolved in water at $10 \mathrm{mg} / \mathrm{mL}$. BLs composed of 1,2-Distearoyl-sn-glycero-3-phosphocholine (DSPC) (Avanti Polar Lipids, Inc., Alabaster, Alabama, USA) and 1,2-distearoyl-sn-glycero-3-phosphatidylethanolaminemethoxy-polyethyleneglycol (DSPE-PEG (2k)-OMe) (NOF Corporation, Tokyo, Japan) $(94: 6(\mathrm{~m} / \mathrm{m}))$ were prepared according to previous reports at $1 \mathrm{mg} / \mathrm{mL}$ in terms of their lipids $[16,17,20,21]$. To encapsulate the perfluoropropane gas into BLs, vials were vortexed in a sonicated bath (BRANSONIC B1200, Emerson Electric Co., St. Louis, MO, USA). The physicochemical properties of BLs were determined with Zetasizer Nano ZS (Malvern Instruments, Worcestershire, UK). 


\section{Ultrasound system and conditions}

Ultrasound was irradiated to the whole brain of mice and rats by SonoPore KTAP-4000 (Nepa Gene Co., Ltd., Chiba, Japan) with 20 mm-diameter probe KP-S20 (Nepa Gene Co., Ltd.). The parameters used were as follows: frequency, $1.045 \mathrm{MHz}$; intensity, 1.0 $\mathrm{W} / \mathrm{cm}^{2}$; duty ratio, $50 \%$; burst rate, $10 \mathrm{~Hz}$; duration, $60 \mathrm{sec}$. For evaluation of the another conditions, the duration $(10,30$ and $120 \mathrm{sec})$ and intensity $\left(0.25\right.$ and $\left.0.5 \mathrm{~W} / \mathrm{cm}^{2}\right)$ were varied.

\section{Brain microdialysis}

Three types of mixed anesthetic agents [22] composed of $0.3 \mathrm{mg} / \mathrm{kg}$ of medetomidine, $4.0 \mathrm{mg} / \mathrm{kg}$ of midazolam and $5.0 \mathrm{mg} / \mathrm{kg}$ of butorphanol (i.p.) were used for the mice, and ethyl carbamate $(1.5 \mathrm{~g} / \mathrm{kg}$, i.p.) was used for the rats. Both mice and rats were fixed with a stereotaxic instrument SR-5R (Narishige Scientific Instrument Lab., Tokyo, Japan). The microdialysis probe A-I-4-02 (Eicom Corporation, Kyoto, Japan) was inserted into the mouse brain (at a coordinate of $A,+2.2 \mathrm{~mm} ; L,+1.0 \mathrm{~mm} ; H,-3.0 \mathrm{~mm}$ from the bregma), and the microdialysis probe CX-I-10-04 (Eicom Corporation) was inserted into the rat brain (at a coordinate of $A,+0.6 \mathrm{~mm} ; L,+5.0 \mathrm{~mm} ; H,-7.0 \mathrm{~mm}$ from the bregma). Each microdialysis probe consists of regenerated cellulose membrane with a molecular weight cut-off of 50,000 Da, and recovery ratios from standard solution of $8.17 \pm 0.67 \%(5-F U, A-I-4-02$ probe, $n=3)$ and $16.2 \pm 0.4 \%$ (ascorbic acid, CX-I-10-04 probe, $n=3)$. Artificial cerebrospinal fluid $(\mathrm{aCSF})$ was used as perfusate at a flow rate of $2 \mu \mathrm{L} / \mathrm{min}$.

\section{Experimental schedules}

The experimental schedule is shown in Scheme 1. Mice and rats were stabilized for $2 \mathrm{~h}$ after insertion of the microdialysis probes into the brain. Then drugs and BLs were 
injected i.v. (100 mg/kg of 5-FU and $7.4 \mathrm{mg} / \mathrm{kg}$ of BLs) or i.p. (50 mg/kg of ascorbic acid), and immediately the brain was subjected to ultrasound. Drug administration, BLs administration and ultrasound irradiation was performed within $5 \mathrm{~min}$ in this order. The time at which the ultrasound irradiation ended was set to $0 \mathrm{~min}$, and the dialysates were collected for $300 \mathrm{~min}$ in intervals of $15 \mathrm{~min}(0-180 \mathrm{~min})$ and $30 \mathrm{~min}(180-300 \mathrm{~min})$.

For evaluation for another conditions of BLs administration and ultrasound irradiation, $100 \mathrm{mg} / \mathrm{kg}$ of $5-\mathrm{FU}$ was used. The following three parameters were examined: the dose of BLs administration, the duration of the ultrasound irradiation and the ultrasound intensity. When one parameter was changed, the other two parameters remained unchanged. Four doses of BLs $(0.925,1.85,3.7$ and $7.4 \mathrm{mg} / \mathrm{kg})$, four durations of ultrasound irradiation $(10,30,60$ and $120 \mathrm{sec})$ and three intensities of ultrasound $\left(0.25,0.5\right.$ and $\left.1 \mathrm{~W} / \mathrm{cm}^{2}\right)$ were selected for the evaluation. The dialysates were collected for $180 \mathrm{~min}$ in 15 -min intervals.

\section{HPLC conditions for determination of 5-FU}

The dialysates from the mouse brain were applied to the high-performance liquid chromatography (HPLC) system for the determination of 5-FU [23]. The HPLC system consisted of an LC-10ADvp pump (Shimadzu Corporation, Kyoto, Japan), a 7725i injector (Rheodyne Inc., Cotati, CA, USA) with a $20 \mu \mathrm{L}$ sample loop, a Wakopak Handy-ODS column (150 mm × $4.6 \mathrm{~mm}$, i.d.; Wako Pure Chemical Industries, Ltd.), an SPD-10Avp ultraviolet detector (detection wavelength, $266 \mathrm{~nm}$; Shimadzu Corporation). 5-FU was separated in $10 \mathrm{mM}$ sodium acetate buffer $(\mathrm{pH} 4.0)$ at a flow rate of 0.7 $\mathrm{mL} / \mathrm{min}$. 


\section{HPLC conditions for determination of ascorbic acid}

The dialysates from the rat brain were doubly dissolved and applied to two HPLC systems for measuring concentration and antioxidant capacity of ascorbic acid. Ascorbic acid concentrations in the dialysates were determined with an HPLC system consisted of an LC-10AT pump (Shimadzu Corporation), a 7125 injector (Rheodyne Inc.) with a $20 \mu \mathrm{L}$ sample loop, a Shodex Asahipak NH2-50 4E column $(250 \times 4.6 \mathrm{~mm}$, i.d.; Showa Denko K.K., Tokyo, Japan), an SPD-10Avp ultraviolet detector (detection wavelength, $254 \mathrm{~nm}$; Shimadzu Corporation). Ascorbic acid was separated in a mixture of $20 \%$ sodium phosphate buffer and $80 \%$ acetonitrile at a flow rate of $1.0 \mathrm{~mL} / \mathrm{min}$.

\section{HPLC conditions for SMFIA method}

The antioxidant capacity of ascorbic acid in the dialysates was measured using the SMFIA method. An HPLC system for the SMFIA was designed as described in a previous report [19]. The antioxidant capacity was presented as the percentages of the scavenged chemiluminescences in the reaction of luminol and reactive oxygen species.

\section{Statistical analysis}

Statistical analysis was performed using JMP ${ }^{\circledR}$ Pro version 11.2.0 (SAS Institute Inc., Cary, NC, USA). $P<0.05$ was defined as significantly different.

\section{Results}

\section{Physicochemical properties of BLs}

The mean diameter and zeta-potential of BLs were $330 \pm 47.0 \mathrm{~nm}$ and $-2.45 \pm 1.51 \mathrm{mV}$, respectively $(n=3)$. 


\section{Time-course evaluation of ultrasound-induced drug delivery into the brain}

\section{Time-course evaluation with 5-FU}

We first confirmed whether microdialysis is useful for evaluation of ultrasound-induced drug delivery into the brain. Figure 1a shows the time-course profiles of 5-FU concentration in dialysates perfused through the mouse brain. In the group treated with both BLs administration and ultrasound irradiation, the 5-FU concentration reached a peak $(1.28 \pm 0.30 \mu \mathrm{g} / \mathrm{mL})$ from $0-15 \mathrm{~min}$ and 5-FU was detected for $150 \mathrm{~min}$ after ultrasound irradiation. Other groups had lower peak concentrations of 5-FU (the group without any stimulations, $0.196 \pm 0.059 \mu \mathrm{g} / \mathrm{mL}$; the group with BLs administration, $0.830 \pm 0.723 \mu \mathrm{g} / \mathrm{mL}$; the group with ultrasound irradiation, $0.397 \pm 0.353 \mu \mathrm{g} / \mathrm{mL}$ ) and shorter continuance of 5-FU recovery after ultrasound irradiation (the group without any stimulations, $60 \mathrm{~min}$; the group with BLs administration, $60 \mathrm{~min}$; the group with ultrasound irradiation, $75 \mathrm{~min}$ ) than the group treated with both BLs administration and ultrasound irradiation. The area under the curves (AUCs) of the time-course profiles are shown in Figure 1b. The AUC of the group with BLs administration and ultrasound irradiation $(63.8 \pm 25.8 \mu \mathrm{g} \cdot \mathrm{min} / \mathrm{mL})$ was significantly $(P<0.05)$ higher than the AUCs of any other groups (the group without any stimulations, $7.51 \pm 1.75 \mu \mathrm{g} \cdot \mathrm{min} / \mathrm{mL}$; the group with BLs administration, $14.6 \pm 10.1 \mu \mathrm{g} \cdot \mathrm{min} / \mathrm{mL}$; the group with ultrasound irradiation, $11.7 \pm 14.1 \mu \mathrm{g} \cdot \mathrm{min} / \mathrm{mL}$ ). Other pharmacokinetic parameters of 5-FU in the brain are shown in Table 1.

\section{Time-course evaluation with ascorbic acid}

To support the result obtained with 5-FU, we examined the time-course profiles using a different species, rats, and a different drug, ascorbic acid. Figure 2a shows the timecourse profiles of ascorbic acid concentration in the dialysates perfused through the rat 
brain. In the group treated with both BLs administration and ultrasound irradiation, the peak concentration of ascorbic acid $(2.01 \pm 0.71 \mu \mathrm{g} / \mathrm{mL})$ was observed from 15-30 min after ultrasound irradiation. The AUC in the group with BLs administration and ultrasound irradiation $(152 \pm 43.1 \mu \mathrm{g} \cdot \mathrm{min} / \mathrm{mL})$ was significantly $(P<0.01)$ higher than the AUCs in the other groups (the group without any stimulation, $17.0 \pm 16.0 \mu \mathrm{g} \cdot$ $\min / \mathrm{mL}$; the group with BLs administration, $11.0 \pm 5.18 \mu \mathrm{g} \cdot \mathrm{min} / \mathrm{mL}$; the group with ultrasound irradiation, $9.50 \pm 2.25 \mu \mathrm{g} \cdot \mathrm{min} / \mathrm{mL}$ ) (Figure $2 \mathrm{~b}$ ). Other pharmacokinetic parameters of ascorbic acid in the brain are shown in Table 2.

Relationship between the pharmacokinetics and pharmacodynamics: Antioxidant capacity of dialysates from the rat brain

Figure 3 shows the time-course profiles of the antioxidant capacity in dialysates perfused through the rat brain. In the group administered BLs and ultrasound irradiation, the peak antioxidant capacity $(76.9 \pm 5.02 \%)$ was observed from $15-30 \mathrm{~min}$. The other groups showed antioxidant capacity of less than $10 \%$.

\section{Effect of BLs and ultrasound for targeting 5-FU delivery into the brain}

\section{Dose of BLs administration}

The effect of BLs on ultrasound-induced drug delivery into the brain was examined by at BLs doses of $0.925,1.85,3.7$, and $7.4 \mathrm{mg} / \mathrm{kg}$ in terms of their lipids. Data are expressed as the time-course profiles from 0 to $180 \mathrm{~min}$ (Figure $4 \mathrm{a}$ ), and the peak concentrations of 5 -FU were $1.02 \pm 0.04 \mu \mathrm{g} / \mathrm{mL}(0.925 \mathrm{mg} / \mathrm{kg}$ group $), 1.15 \pm 0.19$ $\mu \mathrm{g} / \mathrm{mL}$ (1.85 mg/kg group), $1.43 \pm 0.18 \mu \mathrm{g} / \mathrm{mL}$ ( $3.7 \mathrm{mg} / \mathrm{kg}$ group), and $1.89 \pm 0.26$ $\mu \mathrm{g} / \mathrm{mL}$ ( $7.4 \mathrm{mg} / \mathrm{kg}$ group). The AUC from the time-course profile of the $7.4 \mathrm{mg} / \mathrm{kg}$ 
group $(65.9 \pm 20.1 \mu \mathrm{g} \cdot \mathrm{min} / \mathrm{mL})$ was significantly $(P<0.05)$ higher than that from the $0.925 \mathrm{mg} / \mathrm{kg}$ group $(27.2 \pm 7.31 \mu \mathrm{g} \cdot \mathrm{min} / \mathrm{mL})$ (Figure $4 \mathrm{~b})$.

\section{Duration of ultrasound irradiation}

The effect of ultrasound on drug delivery into the brain was examined by varying the duration and intensity of the ultrasound irradiation. The duration of ultrasound irradiation was set to $10,30,60$, and $120 \mathrm{sec}$, and the time-course profiles of 5-FU in each group was examined (Figure 5a). The group with $60 \mathrm{sec}$ of ultrasound irradiation showed the highest peak concentration $(1.63 \pm 0.26 \mu \mathrm{g} / \mathrm{mL})$ followed by the groups irradiated for $30 \mathrm{sec}(1.46 \pm 0.51 \mu \mathrm{g} / \mathrm{mL}), 120 \mathrm{sec}(1.40 \pm 0.60 \mu \mathrm{g} / \mathrm{mL})$, and $10 \mathrm{sec}$ $(1.03 \pm 0.55 \mu \mathrm{g} / \mathrm{mL})$. The AUCs from the time-course profiles for each group were not significantly different (Figure 5b).

\section{Ultrasound intensity}

The ultrasound intensities were set to $0.25,0.5$, and $1 \mathrm{~W} / \mathrm{cm}^{2}$. Figure 6 a shows the timecourse profiles of 5-FU in each group, and the peak concentrations were all approximately $1.2 \mu \mathrm{g} / \mathrm{mL}\left(0.25 \mathrm{~W} / \mathrm{cm}^{2}\right.$ group, $1.17 \pm 0.63 \mu \mathrm{g} / \mathrm{mL} ; 0.5 \mathrm{~W} / \mathrm{cm}^{2}$ group, $1.21 \pm 0.36 \mu \mathrm{g} / \mathrm{mL} ; 1 \mathrm{~W} / \mathrm{cm}^{2}$ group, $\left.1.21 \pm 0.29 \mu \mathrm{g} / \mathrm{mL}\right)$. Additionally, there were no significant differences among their AUCs (Figure 6b).

\section{Discussion}

In this study, we evaluated the detailed pharmacokinetic profiles of two hydrophilic drugs in the brain after delivery with BLs and ultrasound irradiation. First, we used 5FU as a model drug and observed its time-course brain delivery in mice. The highest peak concentration of 5-FU in the dialysate was observed when BLs administration and ultrasound irradiation were combined (Figure 1a). The AUC from the time-course profile of the group subject to BLs administration and ultrasound irradiation was 
significantly $(P<0.05)$ higher than the AUCs from the other groups (Figure $1 \mathrm{~b}$ ). We also evaluated the relationship between the pharmacokinetics and pharmacodynamics for ascorbic acid using microdialysis in the rat brain. The time-course profiles of the antioxidant capacity in the dialysates (Figure 3) corresponded to those where the concentration of ascorbic acid was varied (Figure 2a). Moreover, the result from the time-course profiles versus ascorbic acid concentration (Figure 2a) supports the result with 5-FU (Figure 1a); both 5-FU and ascorbic acid concentrations reached a peak within 30 min after ultrasound irradiation and then rapidly decreased. The difference of pharmacokinetics between 5-FU and ascorbic acid with ultrasound irradiation is not clear still (Figures 1 and 2). Thus, the pharmacokinetics (Figure 2) and pharmacodynamics (Figure 3) for ascorbic acid in the brain were in agreement with each other, and the pharmacokinetics of ascorbic acid (Figure 2) and 5-FU (Figure 1) in the brain were also similar. Moreover, these results are congruent with the report of Yang et al. [12], which showed that the BPA concentration in the dialysates reached a peak after 30 min of ultrasound irradiation to the rat brain. Therefore, we succeeded in clarifying the time-course delivery of hydrophilic drugs into the brain using a microdialysis method by the ultrasound-responsive nanobubbles.

Then, we varied the dose of BLs (Figure 4), and the duration (Figure 5) and intensity (Figure 6) of the ultrasound irradiation. When the dose of BLs was varied from 0.925 to $7.4 \mathrm{mg} / \mathrm{kg}$, the peak concentrations in the time-course profiles of 5-FU (Figure 4a) and their AUCs (Figure 4b) increased in a dose-dependent manner. Additionally, a significant difference was observed between the AUCs of the $0.925 \mathrm{mg} / \mathrm{kg}$ group and the $7.4 \mathrm{mg} / \mathrm{kg}$ group (Figure $4 \mathrm{~b}$ ). Song et al. reported that the volume of microbubbles is the most relevant factor involved in the BBB disruption [24]. In their report, the concentration of Evans blue dye in brain sections increased in a manner dependent on 
the volume of the microbubbles. Although the diameter is different between the microbubbles and the BLs, our result is corresponding the report by Song et al. Therefore, the brain microdialysis would be a useful method to evaluate the pharmacokinetics of the hydrophilic drugs after the brain delivery using bubble formulations and ultrasound irradiation.

On the other hand, when the duration of the ultrasound irradiation was varied from 10 to $120 \mathrm{sec}$, the peak concentrations in the time-course profiles of 5-FU did not correspond to the durations of ultrasound irradiation (Figure 5a). The AUCs of the groups exposed to 10 to $60 \mathrm{sec}$ of ultrasound irradiation increased stepwise, while the AUC of the group exposed to $120 \mathrm{sec}$ was smaller than the group exposed to $60 \mathrm{sec}$. Additionally, there were no significant differences between the AUCs (Figure 5b). When the ultrasound intensity was varied from 0.25 to $1 \mathrm{~W} / \mathrm{cm}^{2}$, the time-course profiles for 5-FU (Figure 6a) and the AUCs (Figure 6b) of the three groups were in accordance with each other. These results may not correspond to other reports of the brain delivery using focused ultrasound $[2,3,5,7,25]$. Because we used unfocused ultrasound and sonicated the whole brain, almost all of the BLs in the blood vessel of the brain might have destroyed, and changing conditions of unfocused ultrasound might have little effect for 5-FU delivery into the brain. In the further study using the unfocused ultrasound, therefore, the conditions of the ultrasound irradiation are needed to be considered to control the drug delivery into brain.

In the field of ultrasound-induced drug delivery into the brain, magnetic resonance imaging (MRI) [2,8] or dye staining [9] are commonly used for time-course evaluations of the brain delivery. These methods use contrast agents such as gadolinium or Evans blue, not drugs themselves. The problem is that the pharmacokinetics of these contrast agents must be different from the targeted drugs from the view point of protein 
binding rate, retentivity in the blood, and molecular weight. In this study, however, we showed that the time-course evaluation of the brain delivery of the hydrophilic drugs themselves was possible. The microdialysis probes inserted may have disrupted the BBB. Nevertheless, 5-FU and ascorbic acid without any stimulations were little delivered into the brain (Figures 1 and 2). Thus, we think that histopathological problems may have not influenced on our evaluation results. Additionally, this is the first report to clarify the time-course pharmacokinetics of hydrophilic drugs in the brain after delivering with bubble formulations and ultrasound irradiation. We believe that the evaluation method with microdialysis enables the analysis for pharmacokinetics of more and more hydrophilic drugs after delivered into the brain because microdialysis recovers drugs themselves.

In this study, however, the results indicated that the drug continuance remaining in the brain was short and the drug concentration rapidly decreased as shown in the time-course profiles (Figures 1 and 2). Drugs delivered into the brain may flow out to the cerebrospinal fluid by the bulk flow. In addition, this phenomenon may also be explained by the fact that the BBB was not closed immediately [2], and drugs in the brain may have leaked out into the blood vessels. This hypothesis corresponds with the report by Negishi et al. that proposes the BBB might be open to some extent for up to $24 \mathrm{~h}$ after the ultrasound irradiation under their experimental conditions [9]. If the BBB closes immediately, drugs are expected to remain in the brain for a longer period. Additionally, it is necessary to control the BBB opening after drug delivery into the brain for safety and effectiveness. We believe that microdialysis enables the easy collection of more detailed information under various conditions of bubble formulations and ultrasound. Using the evaluation method with microdialysis, we will attempt to 
minimise the duration of the BBB opening for safe and efficient brain delivery of drugs using focused and/or unfocused ultrasound in the future.

\section{Conclusions}

We succeeded in clarifying the time-course pharmacokinetics of the two hydrophilic drugs in the brain after the delivery with the ultrasound-responsive BLs and ultrasound irradiation using mice and rats. 
Acknowledgements

This work was supported by JSPS KAKENHI Grant Number 16K18862, the Sasakawa Scientific Research Grant from the Japan Science Society, the Shin-Nihon Foundation of Advanced Medical Research, and Takeda Science Foundation. 


\section{References:}

[1] Fumoto S, Kawakami S. Combination of nanoparticles with physical stimuli toward cancer therapy. Biol. Pharm. Bull. 2014;37:212-216.

[2] Hynynen K, McDannold N, Vykhodtseva N, et al. Noninvasive MR imagingguided focal opening of the blood-brain barrier in rabbits. Radiology. 2001;220:640-646.

[3] Kinoshita M, McDannold N, Jolesz FA, et al. Noninvasive localized delivery of Herceptin to the mouse brain by MRI-guided focused ultrasound-induced bloodbrain barrier disruption. Proc. Natl. Acad. Sci. U. S. A. 2006;103:11719-11723.

[4] Sheikov N, McDannold N, Sharma S, et al. Effect of focused ultrasound applied with an ultrasound contrast agent on the tight junctional integrity of the brain microvascular endothelium. Ultrasound Med. Biol. 2008;34:1093-1104.

[5] Liu H-L, Hua M-Y, Chen P-Y, et al. Blood-brain barrier disruption with focused ultrasound enhances delivery of chemotherapeutic drugs for glioblastoma treatment. Radiology. 2010;255:415-425.

[6] Chen Y-C, Chiang C-F, Wu S-K, et al. Targeting microbubbles-carrying TGF $\beta 1$ inhibitor combined with ultrasound sonication induce BBB/BTB disruption to enhance nanomedicine treatment for brain tumors. J. Control. Release. 2015;211:53-62.

[7] Treat LH, McDannold N, Vykhodtseva N, et al. Targeted delivery of doxorubicin to the rat brain at therapeutic levels using MRI-guided focused ultrasound. Int. J. Cancer. 2007;121:901-907.

[8] Aryal M, Vykhodtseva N, Zhang YZ, et al. Multiple treatments with liposomal doxorubicin and ultrasound-induced disruption of blood-tumor and blood-brain barriers improve outcomes in a rat glioma model. J. Control. Release. 2013;169:103-111.

[9] Negishi Y, Yamane M, Kurihara N, et al. Enhancement of blood-brain barrier permeability and delivery of antisense oligonucleotides or plasmid DNA to the brain by the combination of bubble liposomes and high-intensity focused ultrasound. Pharmaceutics. 2015;7:344-362.

[10] Benveniste H. Brain microdialysis. J. Neurochem. 1989;52:1667-1679.

[11] Johansen MJ, Newman RA, Madden T. The use of microdialysis in pharmacokinetics and pharmacodynamics. Pharmacotherapy. 1997;17:464-481. 
[12] Yang FY, Lin YL, Chou FI, et al. Pharmacokinetics of BPA in gliomas with ultrasound induced blood-brain barrier disruption as measured by microdialysis. PLoS One. 2014;9:1-6.

[13] Un K, Kawakami S, Yoshida M, et al. Efficient suppression of murine intracellular adhesion molecule-1 using ultrasound-responsive and mannosemodified lipoplexes inhibits acute hepatic inflammation. Hepatology. 2012;56:259-269.

[14] Un K, Kawakami S, Suzuki R, et al. Development of an ultrasound-responsive and mannose-modified gene carrier for DNA vaccine therapy. Biomaterials. 2010;31:7813-7826.

[15] Abdalkader R, Kawakami S, Unga J, et al. Evaluation of the potential of doxorubicin loaded microbubbles as a theranostic modality using a murine tumor model. Acta Biomater. 2015;19:112-118.

[16] Suzuki R, Oda Y, Omata D, et al. Tumor growth suppression by the combination of nanobubbles and ultrasound. Cancer Sci. 2016;107:217-223.

[17] Nishimura K, Fumoto S, Fuchigami Y, et al. Effective intraperitoneal gene transfection system using nanobubbles and ultrasound irradiation. Drug Deliv.. 2017;24:737-744.

[18] Yuki F, Rie I, Miki K, et al. Warning against co-administration of 3,4methylenedioxymethamphetamine (MDMA) with methamphetamine from the perspective of pharmacokinetic and pharmacodynamic evaluations in rat brain. Eur. J. Pharm. Sci. 2013;49:57-64.

[19] Wada M, Kira M, Nakaji Y, et al. Development of a novel method for monitoring the antioxidative effect of ascorbic acid in rat blood. Food Chem.. 2012;134:546-552.

[20] Suzuki R, Takizawa T, Negishi Y, et al. Gene delivery by combination of novel liposomal bubbles with perfluoropropane and ultrasound. J. Control. Release. 2007;117:130-136.

[21] Abdalkader R, Kawakami S, Unga J, et al. The development of mechanically formed stable nanobubbles intended for sonoporation-mediated gene transfection. Drug Deliv. 2017;24:320-327.

[22] Kawai S, Takagi Y, Kaneko S, et al. Effect of three types of mixed anesthetic agents alternate to ketamine in mice. Exp. Anim. 2011;60:481-487. 
[23] Kawakami S, Horimoto T, Nishida K, et al. Kidney- and site-selective delivery of 5-fluorouracil utilizing the absorption on the kidney surface in rats. Biol. Pharm. Bull.. 2002;25:928-930.

[24] Song K, Fan AC, Hinkle JJ, et al. Microbubble gas volume: A unifying dose parameter in blood-brain barrier opening by focused ultrasound. Theranostics. 2017;7:144-152.

[25] Fan C-H, Liu H-L, Ting C-Y, et al. Submicron-bubble-enhanced focused ultrasound for blood-brain barrier disruption and improved CNS drug delivery. PLoS One. 2014;9:e96327. 


\begin{tabular}{|l|l|l|l|l|}
\hline & 5-FU only & 5-FU + BLs & 5-FU + US & $\begin{array}{l}\text { 5-FU + BLs } \\
+\mathbf{U S}\end{array}$ \\
\hline $\mathbf{T}_{\mathbf{m a x}}, \mathbf{m i n}$ & $17.5 \pm 8.7$ & $12.5 \pm 8.7$ & $12.5 \pm 8.7$ & $17.5 \pm 8.7$ \\
\hline $\mathbf{C}_{\mathbf{m a x}}, \mathbf{n g} / \mathbf{m L}$ & $195.6 \pm 58.5$ & $830.2 \pm 723.4$ & $397.3 \pm 352.6$ & $\begin{array}{l}1275.6 \pm \\
304.3\end{array}$ \\
\hline $\mathbf{t}_{\mathbf{1} / \mathbf{2}}, \mathbf{m i n}$ & & & & $27.3 \pm 10.0$ \\
\hline $\mathbf{A U C}, \boldsymbol{\mu g} \cdot \mathbf{m i n} / \mathbf{m L}$ & $7.51 \pm 1.75$ & $14.6 \pm 10.1$ & $11.7 \pm 14.1$ & $63.8 \pm 25.8$ \\
\hline MRT, $\mathbf{m i n}$ & $39.9 \pm 23.5$ & $16.5 \pm 4.0$ & $19.4 \pm 7.9$ & $39.5 \pm 14.4$ \\
\hline
\end{tabular}

Table 1. Pharmacokinetic parameters of 5-FU in the brain. Each group contained threetime trials, and data are expressed as the mean \pm S.D. 


\begin{tabular}{|l|l|l|l|l|}
\hline & $\begin{array}{l}\text { Ascorbic } \\
\text { acid only }\end{array}$ & $\begin{array}{l}\text { Ascorbic acid } \\
+ \text { BLs }\end{array}$ & $\begin{array}{l}\text { Ascorbic } \\
\text { acid + US }\end{array}$ & $\begin{array}{l}\text { Ascorbic } \\
\text { acid + BLs } \\
\text { US }\end{array}$ \\
\hline $\mathbf{T}_{\mathbf{m a x}}, \mathbf{m i n}$ & $15.0 \pm 13.0$ & $57.5 \pm 48.2$ & $122.5 \pm 86.6$ & $22.5 \pm 0.0$ \\
\hline $\mathbf{C}_{\mathbf{m a x}}, \mathbf{n g} / \mathbf{m L}$ & $219.4 \pm 174.8$ & $128.4 \pm 113.7$ & $132.5 \pm 114.9$ & $\begin{array}{l}2014.3 \pm \\
712.6\end{array}$ \\
\hline $\mathbf{t}_{\mathbf{1} / \mathbf{2}}, \mathbf{m i n}$ & $39.5 \pm 9.9$ & $54.5 \pm 9.2$ & $81.5 \pm 34.0$ & $45.9 \pm 0.9$ \\
\hline $\mathbf{A U C}, \boldsymbol{\mu g} \cdot \mathbf{m i n} / \mathbf{m L}$ & $17.0 \pm 16.0$ & $11.1 \pm 5.2$ & $9.50 \pm 2.25$ & $151.7 \pm 43.1$ \\
\hline MRT, $\mathbf{m i n}$ & $56.9 \pm 14.2$ & $78.6 \pm 13.3$ & $117.6 \pm 49.0$ & $66.2 \pm 1.2$ \\
\hline
\end{tabular}

Table 2. Pharmacokinetic parameters of ascorbic acid in the brain. Each group contained three-time trials, and data are expressed as the mean \pm S.D. 
Figure captions:

Scheme 1. Experimental schedule of microdialysis. After operation and insertion of microdialysis probes, mice and rats were stabilised for $2 \mathrm{~h}$ under the flow of perfusate. During the last 15 min of the stabilization, 'pre-administration' dialysate was collected. After the stabilization, drug administration, BLs administration and ultrasound irradiation was performed within 5 min in this order. The moment when ultrasound irradiation ended was set to $0 \mathrm{~min}$, and dialysates was collected for $300 \mathrm{~min}$ or $180 \mathrm{~min}$. Dialysates were collected in 15-min intervals from 0-180 min and 30-min intervals from $180-300 \mathrm{~min}$.

Figure 1. Time-course profiles of 5-fluorouracil (5-FU) concentrations in the dialysates from the mouse brain (a) and their area under the curves (AUCs) (b). Each group contained three-time trials, and data are expressed as the mean \pm S.D. ${ }^{*} P<0.05$ and ** $P<0.01$ by Tukey's test. US: ultrasound.

Figure 2. Time-course profiles of ascorbic acid concentrations in the dialysates from the rat brain (a) and their area under the curves (AUCs) (b). Each group contained threetime trials, and data are expressed as the mean \pm S.D. $* P<0.05$ and $* * P<0.01$ by Tukey's test. US: ultrasound.

Figure 3. Time-course profiles of antioxidant capacity of the dialysates from the rat brain. Each group contained three-time trials, and data are expressed as the mean \pm S.D. US: ultrasound.

Figure 4. Time-course profiles of 5-fluorouracil (5-FU) concentrations in the dialysates from the mouse brain (a) and their area under the curves (AUCs) (b). The groups were divided based on the four doses of BLs administration: $0.925,1.85,3.7$, and $7.4 \mathrm{mg} / \mathrm{kg}$. 
Each group contains three-time trials, and data are expressed as the mean \pm S.D. ${ }^{*} P<0.05$ by Tukey's test. US: ultrasound.

Figure 5. Time-course profiles of 5-fluorouracil (5-FU) concentrations in the dialysates from the mouse brain (a) and their area under the curves (AUCs) (b). The groups were divided based on the four durations of the ultrasound irradiation: 10, 30, 60 and $120 \mathrm{sec}$. Each group contained three-time trials, and data are expressed as the mean \pm S.D. There were no significant differences between the AUCs. US: ultrasound.

Figure 6. Time-course profiles of 5-fluorouracil (5-FU) concentrations in the dialysates from the mouse brain (a) and their area under the curves (AUCs) (b). The groups were divided based on the four intensities of the ultrasound: $0.25,0.5$ and $1 \mathrm{~W} / \mathrm{cm}^{2}$. Each group contained three-time trials, and data are expressed as the mean \pm S.D. There were no significant differences between the AUCs. US: ultrasound. 
Scheme 1

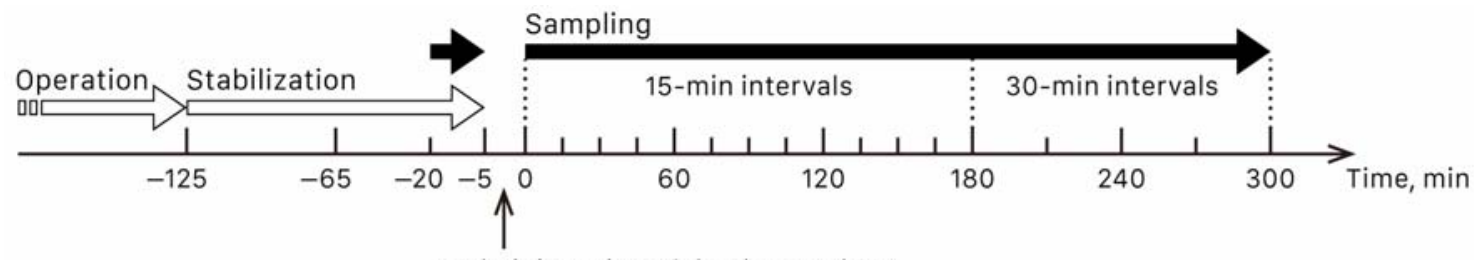

- Administration of the drug and BLS

- Ultrasound irradiation 
Figure 1

(a)

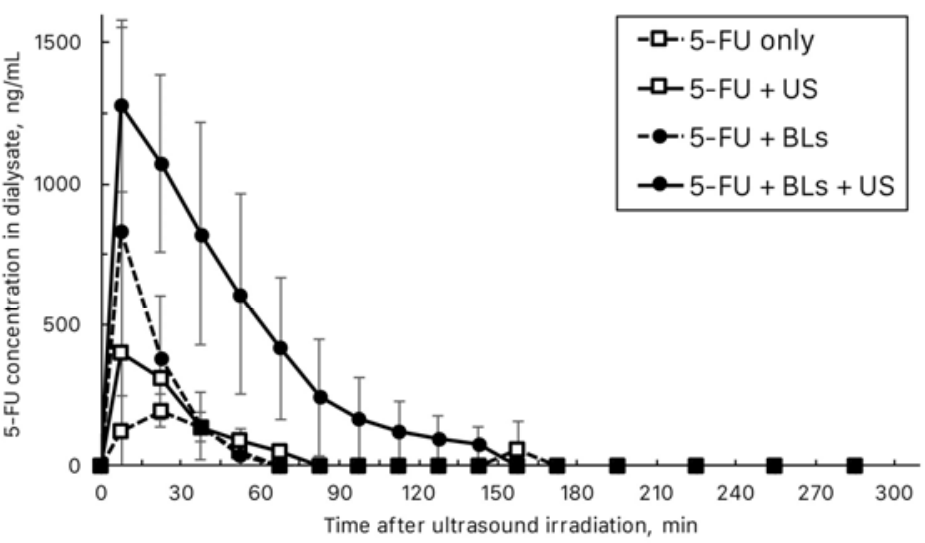

(b)

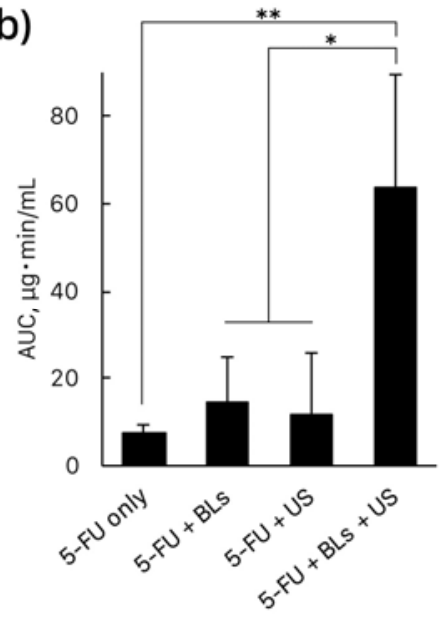

Figure 2

(a)

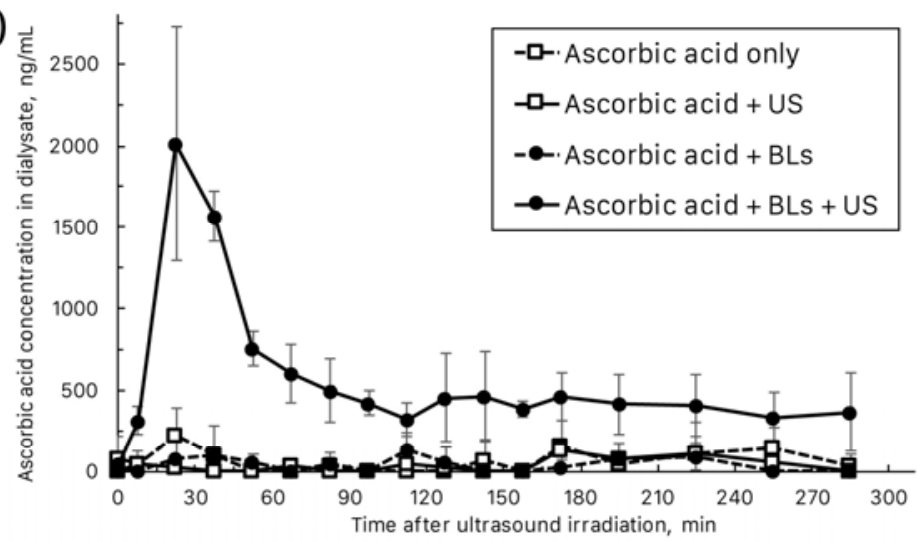

(b)

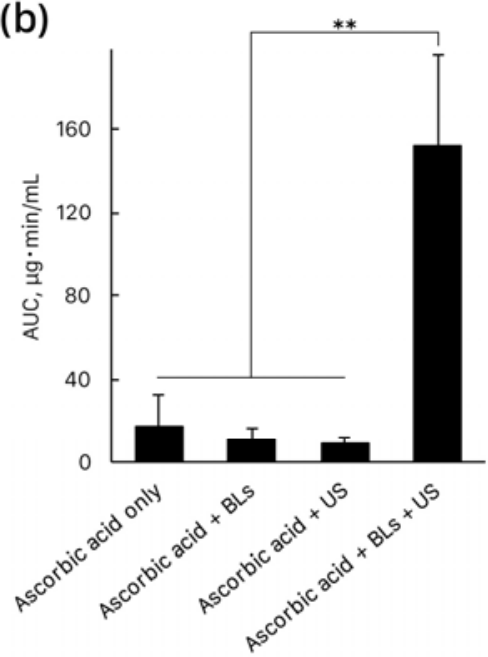


Figure 3

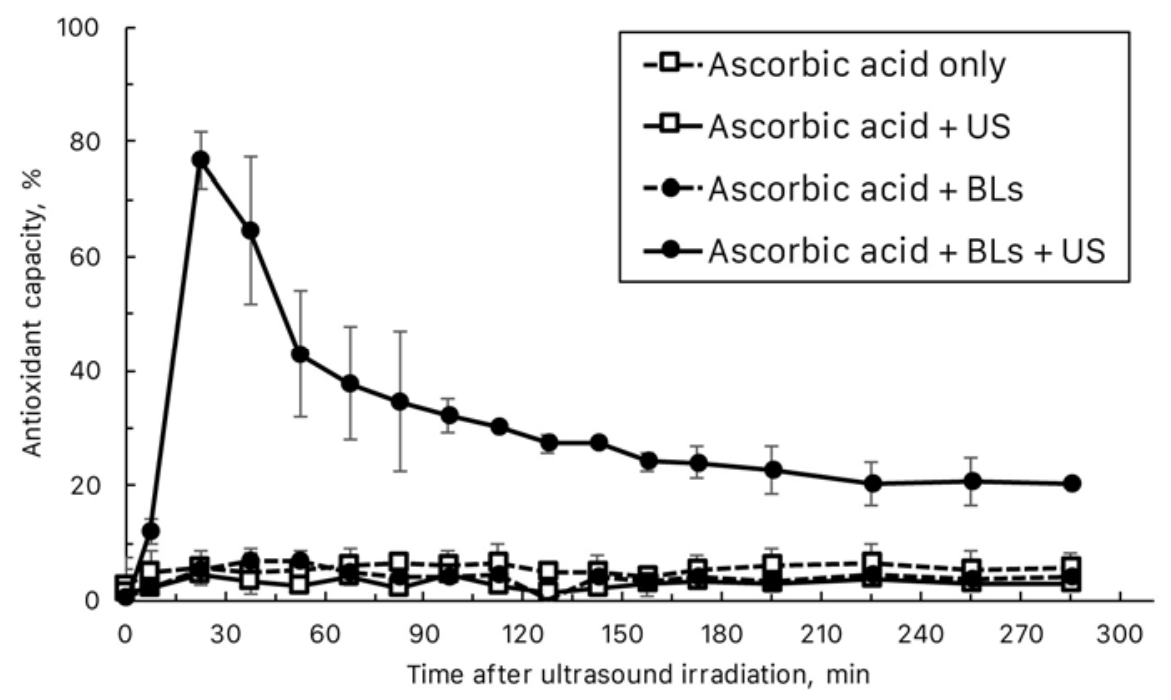

Figure 4
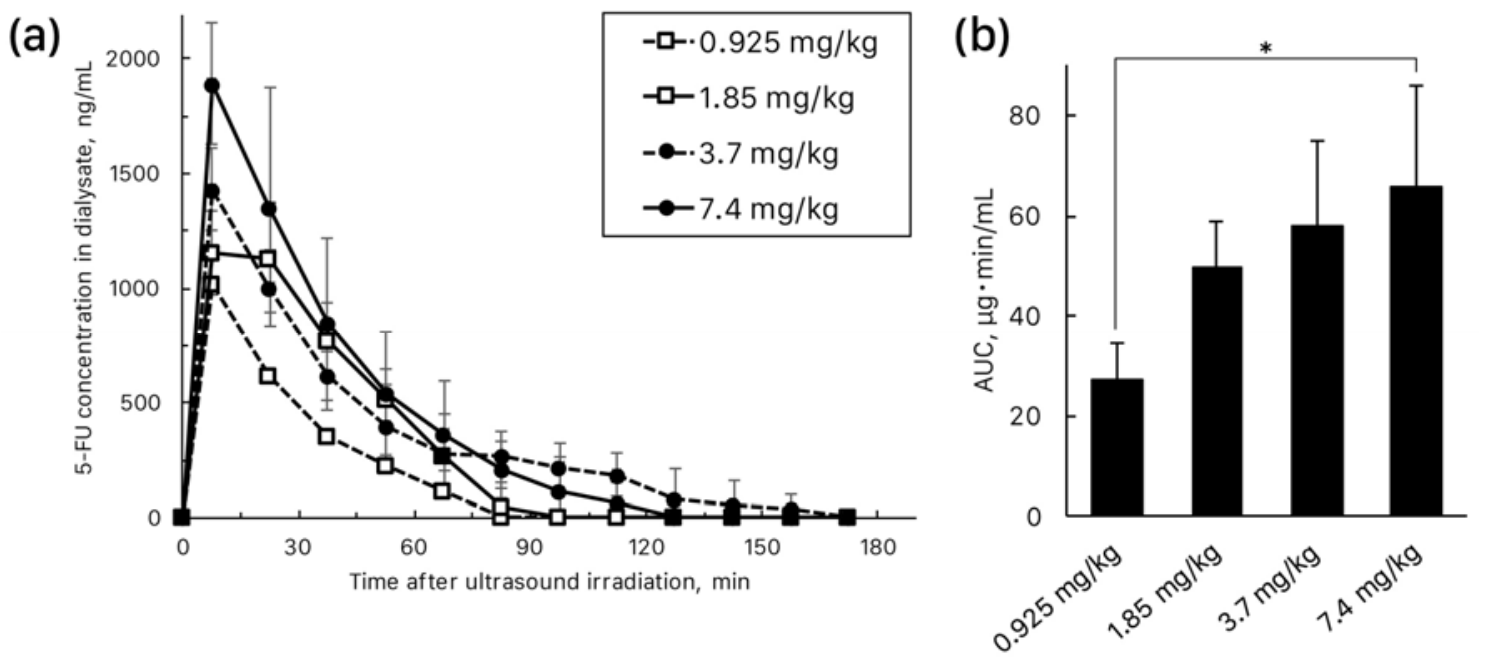
Figure 5

(a)

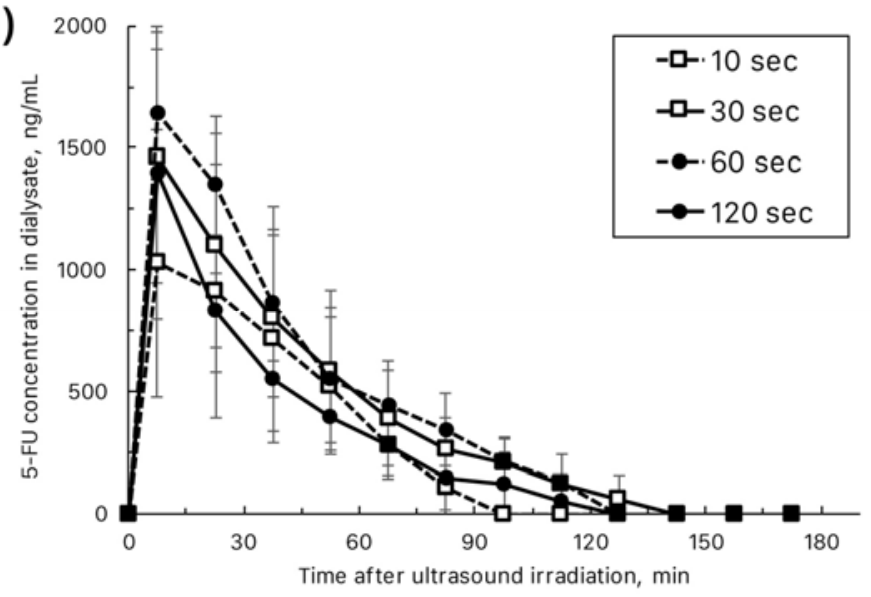

(b)

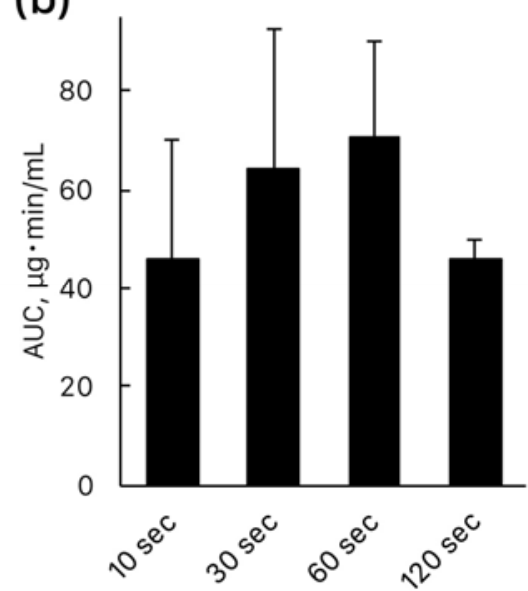

Figure 6

(a)

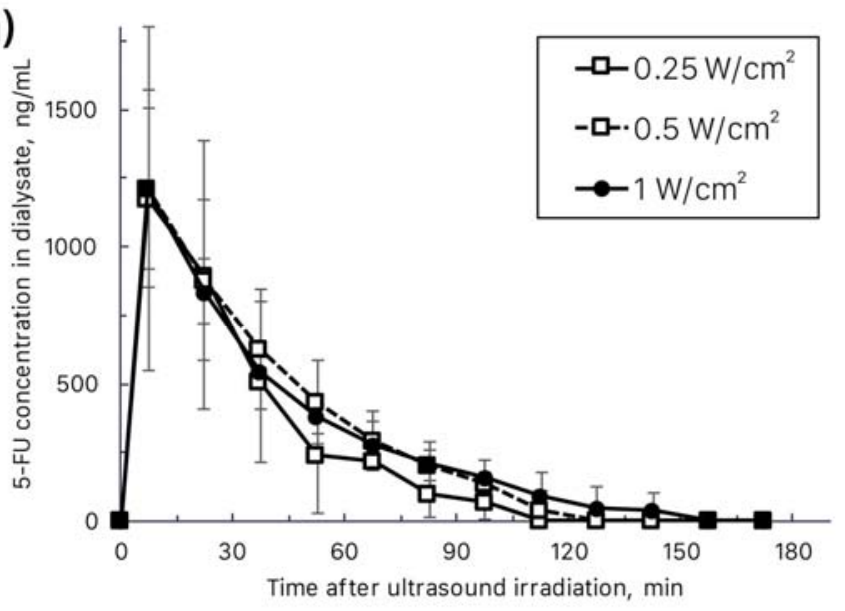

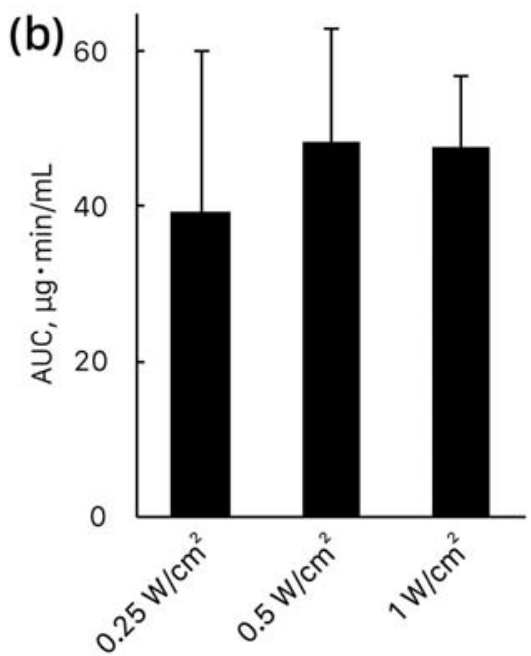

